\title{
Wearable and Ambient Sensing for Well-being and Emotional Awareness in the Smart Workplace
}

\author{
Katrin Hänsel \\ Queen Mary University of London \\ London, UK \\ k.hansel@qmul.ac.uk
}

\section{ACM Classification Keywords}

H.5.m Information interfaces and presentation (e.g., HCI): Miscellaneous; J.3 Computer Applications: Life And Medical Science - Consumer Health

\section{Author Keywords \\ wearable technology, ambient intelligence, well-being}

\section{PROBLEM DOMAIN}

Wearable, sensor-equipped devices - often referred to as wearables - became increasingly public accessible during the last years. Health and fitness wearables offer ubiquitous and continuous sensing of various aspects of our lives and help us reflect and learn about ourselves. The rising social acceptance of body-worn technology is also a driver for the increasing adoption of wearables on the consumer level - and especially the fitness and healthcare sector is booming [1]. Consumer surveys discovered that users expect wearables to help them live longer and happier lives [6].

In our modern, hectic lives, stress becomes an increasing factor diminishing our health. Chronic stress is highly related to cardiovascular diseases [24], cancer [12] and a shortening of lifespan [5]. There is also indication that high stress levels are related to decreased happiness in life [22]. The increasing workload and pressure at the workplace can add to this stress. The prevention of long-term stress and the promotion of wellbeing, hence, can help us to live longer, healthier and happier lives.

I am especially interested in using emerging wearable sensing data to detect stress and emotions in the office workplace, where stress is very common and negatively influences the well-being and atmosphere [14]. I want to explore new ubiquitous, wearable sensing approaches to not just infer current stress and emotional states of the wearer, but also detect personal influencing factors, such as environmental and social

Permission to make digital or hard copies of all or part of this work for personal or classroom use is granted without fee provided that copies are not made or distributed for profit or commercial advantage and that copies bear this notice and the full citation on the first page. Copyrights for components of this work owned by others than the author(s) must be honored. Abstracting with credit is permitted. To copy otherwise, or republish, to post on servers or to redistribute to lists, requires prior specific permission and/or a fee. Request permissions from Permissions@acm.org.

Ubicomp/ISWC'16 Adjunct, September 12 - 16, 2016, Heidelberg, Germany Copyright is held by the owner/author(s). Publication rights licensed to ACM. ACM 978-1-4503-4462-3/16/09...\$15.00

DOI: http://dx.doi.org/10.1145/2968219.2971360 conditions. Furthermore, I am interested if presenting emotional and stress levels of co-workers in an ethical and privacy conform manner combined with utilising ambient presentation modes and reactive technology could raise awareness and trigger empathy within the workplace.

\section{RELATED WORK}

There has been some work on Ambient Intelligence (AmI) at workplace and wearable technology to detect stress levels and emotional states.

\section{Wearable Technology for Stress Monitoring}

Stress and emotions are very complex processes and states which are influenced by various factors. Negative emotions and stress can influence our well-being. Especially long-term and chronic stress has negative effects on well-being and health [26]. In general, stress is an affective reaction to high demands and challenges. This reaction is called fight or flight effect and can be considered as a positive reaction which helps us to meet the challenges in stressful situations. But if this reaction is present over a longer period of time and becomes chronic, it can cause physical and psychological illnesses. The Circumplex Model of Affect is an established model to classify affective states in a two-dimensional space consisting of an Valence (Positivity) and Arousal (Activeness) axis [20]. Based on this model, stress is classified as a negative, high-arousal affective state.

There are different approaches on sensing stress and emotional states, using remote, mobile or wearable sensing.

\section{Remote Sensing}

There has been research on remotely estimating stress levels of users. One common approach is the use of visual analysis and sensing to extract facial expressions [30]. Webcams have also been utilised to detect the blood flow under the skin, estimate heart rate and making assumptions on mental workload and stress [4]. Pressure-sensitive keyboard and capacitive mouse input at the desk have been used to determine the stress levels of co-workers [8].

While these sensing approaches may be suitable for stationary office work, they might not capture interesting data points happening away from the desk and while interacting with colleagues for instance. 


\section{Mobile Sensing}

There is research on using mobile phone sensing to detect stress and emotional states. MoodScope, which assesses the user's current mood in terms of happiness and activeness, has utilised phone usage data, like calls, sms, location and application usage to correlate emotional states of users [10]. Another project used the mobile phone usage data (like SMS and phone calls), weather data and personal traits of the user to predict stress with a $72 \%$ accuracy [3]. Mobile phone usage data was also used to detect boredom [15] - the affective state opposite stress/excitement on the arousal scale in the in the Circumplex Model of Affect [20]. Their model reached an accuracy of $74.6 \%$ to $82.9 \%$.

The StudentLife project, for example, used mobile phone data of students and correlated it with their academic performance and depression levels [28]. The Affective Diary utilized mobile phone usage data, photos and bluetooth to detect nearby people [23]. They used this data to detect the stressed state of the user and presented the data in a diary format to the user, so they could reflect on their stressed states.

\section{Wearable Sensing}

Wearable sensing offers the benefit of a closeness to the body; this not just provides a higher chance of continuous sensing data compared to mobile phones, which are sometimes left behind, but it also enables biosignal sensing. Biosignals, which can be unobtrusively measured by wearable sensors in a noninvasive manner, can be used to determine current stress and emotional levels [2, 17]. Two very common biosignals are the Heart Rate/Heart Rate Variability and the Electrodermal Activity.

Electrodermal Activity (EDA) describes the changes in the electrical properties of the skin. The most commonly used property is the skin conductance (often called Galvanic Skin Response (GSR)). EDA is linked to emotional and cognitive processes and it is often used for emotional measurements [7, 16]. The easiness to measure the skin conductance, makes it a good tool for wearable technology. Several projects have focused on using skin conductance measurements to sense emotional states and provide visualisations for users to reflect $[23,21]$. Kocielnik et al. evaluated the use of Galvanic Skin Response for detecting stress at workplace and matched the data to calendar data to help the user reflect [9]. They did not present real-time data to the user, though, and they did not intervene in stressful situations. But they found, that users liked to reflect on their stress levels to identify stressors afterwards. Other projects experienced that feedback and real-time interventions on stress levels can act as an additional stressor [11].

Heart Rate Variability (HRV) is a common biosignal to detect different internal states of the body and it is often used to detect arousal states $[25,27]$. The heart of a relaxed, healthy person does not beat regularly, but is influenced by respiration and the autonomous nervous system. This leads to a constant change of the intervals between two heart beats. When the person becomes stressed, the interval between two beats become more and more steady and the variability decreases.
Wearable technology offers the great advantage over mobile phones, that devices can be easily and continuously worn, while personal mobile phones are sometimes left behind at the desk. Furthermore, they open up new data sources like biosignals, which have a long history for being used in research setting to detect arousal and stress states. While devices to measure these used to be mostly expensive and specialised, advances in consumer technology opened up new opportunities on broadly available wearables equipped with heart rate and skin conductance sensors ${ }^{1}$.

\section{Ambient Intelligence}

Ambient Intelligence (AmI) refers to the vision that technology becomes embedded in a smart, unobtrusive environment, which is sensing the user's presence and reacting to it in a context-sensitive way [29]. Ambient Intelligence environments often focus on easing the lives of people through the use of intelligent actions and decisions [13]. This is often linked to ubiquitous and pervasive computing and the unobtrusive embedding of sensing technology in everyday devices to enable the connection of these.

In the past, different research has focused on possible scenarios for AmI in workplace environments [19]. They mostly focused on new interactions or communication techniques, user-adaptive environments for environmental comfort, or personal assistance for the employes. There is only a small part focusing on the personal well-being of the workers, but it mostly addressed thermal and environmental comfort.

A study from Röcker investigated different scenarios for Ambient Intelligence at the workplace [18]. He found, that especially well-being-focused applications were rated with a high 'intention of use' by the participants. This indicates a high chance of user adaption of those applications. But implementing smart technologies which addresses sensitive user data in a shared space like the work environment, demand thoughtful design considerations to secure the privacy of the users. Röcker argued that the discomfort from possible viewers watching public interactions in the AmI environment, could lead to the rejection and failure of the system. The combination of Ambient Intelligence in the semi-public workplace environment and the private feedback of personal wearable devices could address these concerns.

There is high potential to combine Ambient Intelligence with stress-sensing on-body sensors to include knowledge of the user's internal state to the smart environment. The wearable sensors can provide personal indication on emotional levels, which then can be used to generate unobtrusive and contextsensitive feedback within the AmI environment. It is worth investigating if this combination can lead to a gentle way for stress relief in workplace environments.

\section{METHODOLOGICAL APPROACH}

The goal of this doctoral project is the evaluation of suitable wearable and ambient sensing technologies for inferring

\footnotetext{
${ }^{1}$ Examples are the Microsoft Band 2 and the Apple Watch
} 
stress states and influencing factors of users in an shared office environment, with the greater goal to support a collective stress management within working environments through raised awareness.

One key part is the evaluation of suitable wearable and ambient sensing technologies to infer current stress states as well as influencing factors such as environmental, social and work-related factors. This involves the correlation of sensing data with user-provided experience samples, diary notes and questionnaire results. This step builds the basis for further experiments addressing interventions and media to promote a raised collective awareness on influencing stressors and enhanced social connectedness, as well as the impact of such technologies on the co-workers. This step will especially focus on privacy concerns regarding the sensitive and personal data and implications on the social infrastructure in the office environment.

\section{KEY IDEA AND HYPOTHESIS}

The main question I want to investigate in my project, is

"Can ambient and wearable technology help the individual to manage and improve stress and well-being at the workplace by supporting social awareness and connectedness?"

I have identified three objectives I want to focus on to investigate my research question:

- RO1: Evaluate Stress Perceptions and Factors at the Workplace - How do people perceive stress and social/emotional ties at the workplace? How does it affect their well-being?

- RO2: Detecting Stress Factors at Workplaces Using Wearable and Ambient Sensing - How can we detect stress, stress factors, emotional states and social interactions at the workplace by using wearable and ambient sensing?

- RO3: Evaluating Techniques to Positively Influence Stress, Emotional States, and Social Connectedness and Awareness at Workplace Using Wearable and Ambient Feedback - Which ambient and wearable feedback can be used to make the users aware of their own stress and emotion states, as well as their co-workers states, with regards to ethics, privacy and usefulness? How does such feedback influence the workplace awareness and connectedness with regards to a positive workplace atmosphere?

\section{RESEARCH PROGRESS AND PLAN}

The research plan is also divided to address the 3 main research objectives.

Evaluate Stress Perceptions and Factors at the Workplace One part of this objective RO1 is the involvement of users in a study to question them about their perception of stress at the workplace with special regards to their social interaction with co-workers. This part of the project has the aim to get an understanding on how the employees at a workplace perceive stress, what they perceive as influencing factors, and how they usually cope with these. This part of the project is also intended to gain insights on the point-of-view of potential users on the usage of technology and smart feedback from mobile phones, wearables or the ambient environment for supporting the personal stress management. An additional literature study complements this part.

This research objective contributes to the overall research question by assessing the users stance on the subject. It can hence help to design an approach better suited and adapted to the users' needs.

In accordance with this research objective, I prepared an online survey to investigate perceptions of stress and well-being at the workplace and personal techniques to overcome these. The results indicated a general interest in wearable technology and stress management amongst the participants, and confirmed that workplace stress is indeed a prevalent thing amongst the survey participants.

\section{Detecting Stress Factors at Workplaces Using Wearable and Ambient Sensing}

The second part of this project is supposed to look at ways to measure and identify factors influencing stress and well-being by using wearable and ambient sensing. This objective has the aim to measurably identify relationships of influencing factors at workplaces, which lead to stress and effect social connectedness and awareness. It contains an evaluation of appropriate sensors to detect stress, emotions and social interactions as well as environmental factors. These sensors are on one hand wearable and personal, and on the other hand ambient, integrated in the surrounding workplace area. A special focus is on the impact of social interactions with co-workers and how these interactions influence the own well-being.

The sensors identified in this stage will be the basis and data source for suitable interventions and feedback strategies of the third part of the project.

\section{Evaluating Techniques to Positively Influence Stress, Emotional States, and Social Connectedness and Aware- ness at Workplace Using Wearable and Ambient Feed- back}

The last part of the project deals with the design and evaluation of new techniques using wearable and ambient feedback and interventions to raise awareness concerning stress and emotional states, with the aim to reduce stress and increase well-being. This part is strongly related to RO2 and builds upon its findings. A study in a workplace setting shall investigate, how these new strategies can decrease and prevent stress and increase emotional well-being.

\section{Current Stage}

Last year, I conducted an online survey with regards to RO1 and the question on how users perceive and manage stress at the workplace and their opinion on technological support.

In preparation of my research proposal, I contributed to a detailed literature review on the use of wearable technology for health behaviour changes, which is currently under review for the ACM Computing Surveys journal. 


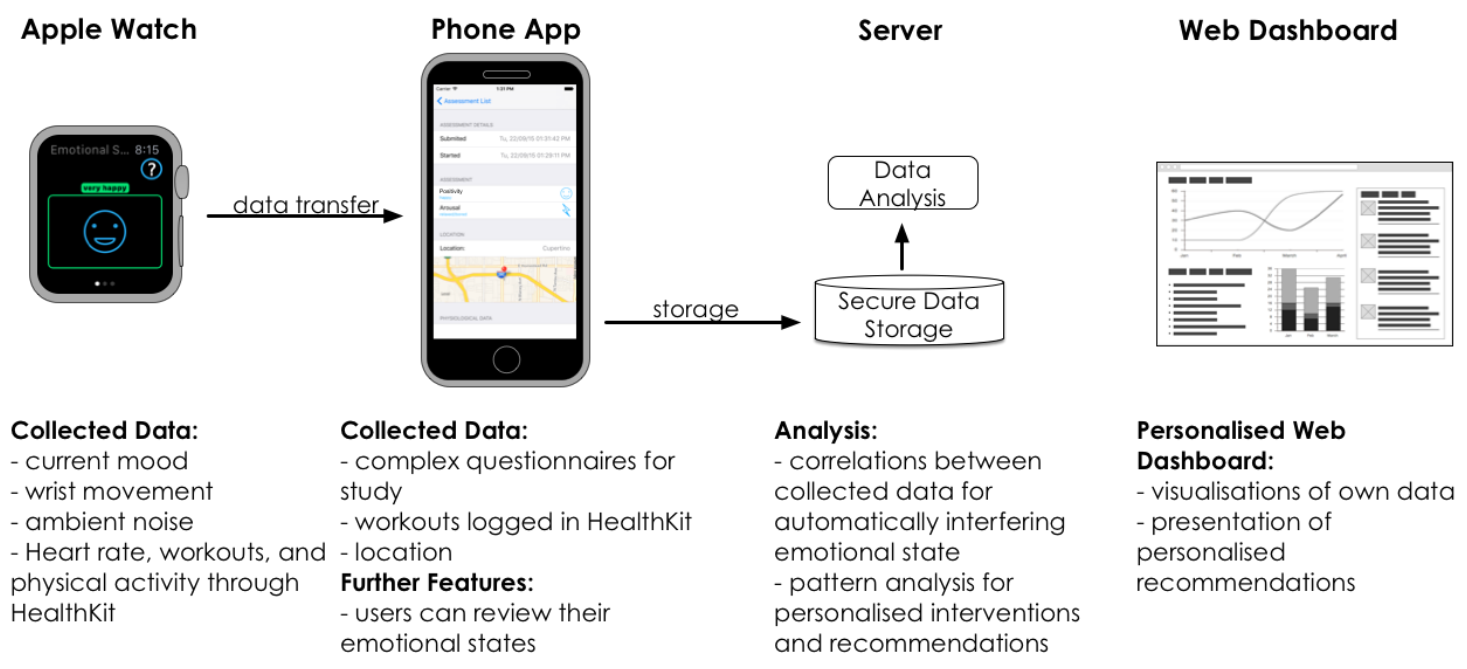

Figure 1. Overview of the Apple Watch/iOS system for emotion/stress experience sample and sensing data collection with the aim of determining the Apple Watch's suitability for inferring current stress states.

I currently evaluate the Apple Watch, as a broadly available consumer Wearable, on the suitability for stress detection. I develop an app for stress self-assessments on the watch and additionally record heart rate features, physical activity, location, ambient noise, to later analyse this data. This data will be used to determine the suitability of the collected sensing data to infer current emotional and stress states. Furthermore, there will be a companion app on the phone presenting additional questionnaires at different study points to gain insight in the user's personal perception of stress. The collected data is securely stored on a web server and accessible from there for the analysis. A related Master's project will focus on the development of an web dashboard extension for presenting the collected experience sample and sensing data to users, with the aim to enhance the interest in the user study and support the self-reflection. An overview can be seen in Figure 1. This step aims to evaluate the Apple Watch as a suitable wearable for unobtrusively detecting stressful states of users. The Apple Watch, as an affordable, broadly available smartwatch with biosignal sensors, could build the data source for detecting stress and triggering interventions and feedback in the future project stages.

Future, but yet just vaguely planned, steps involve the evaluation of ambient sensing strategies for detecting stress influencing factors, such as ambient environment aspects and social interactions. This involves the collaboration with related in-house projects on mobile crowd interaction sensing and ambient sensing for office comfort.

\section{EVALUATION METHODS AND STEPS}

Quantitative and Qualitative methods will be used throughout the project to evaluate different aspects. Evaluation of suitable sensing approaches will focus on statistical analysis and machine learning approaches to develop algorithms and classifiers for the identification of reliable inference of stressed states, automatic detection of social co-worker inter- action and the determination of a personal stress-influencing factors. These developed algorithms will build the basis for the further investigation on different feedback strategies.

For the RO3 and the development of effective ambient feedback strategies to support stress management in the workplace, we will focus on smaller case studies for different approaches and will involve, additionally to the sensing and usage data collection, participant interviews and open-ended questionnaires.

\section{EXPECTED CONTRIBUTIONS IN THE FIELD OF UBIQUI- TOUS COMPUTING}

I intend to make the following contributions with my studies:

1. Identify ubiquitous and unobtrusive wearable and ambient sensors to detect emotions and stress levels of co-workers.

2. Develop an effective feedback strategy, using wearable and ambient feedback, to improve the awareness of stress and emotional states with the aim to improve the social connectedness and well-being of the co-workers.

In the long term, I would like to develop and investigate strategies to support the well-being and social connectedness at the modern workplace by raising emotional awareness thorough technology as a mediator and facilitator.

\section{REFERENCES}

1. ABI Research. Global Wearable Computing Devices, World Market, Forecast: 2013 to 2019.

http://blog.imgtec. com/wp-content/uploads/2014/11/ ABI-Research-global-wearable-computing-devices.png

2. Andrew P Allen, Paul J Kennedy, John F Cryan, Timothy G Dinan, and Gerard Clarke. 2014. Biological and Psychological Markers of Stress in Humans: Focus on the Trier Social Stress Test. Neuroscience and Biobehavioral Reviews 38 (Jan 2014), 94-124. DOI : http://dx.doi.org/10.1016/j .neubiorev.2013.11.005 
3. Andrey Bogomolov, Bruno Lepri, Michela Ferron, Fabio Pianesi, and Alex Sandy Pentland. 2014. Daily Stress Recognition From Mobile Phone Data, Weather Conditions and Individual Traits. In Proceedings of the ACM International Conference on Multimedia (MM'14). 477-486. DOI :

http://dx.doi.org/10.1145/2647868.2654933

4. Frederic Bousefsaf, Choubeila Maaoui, and Alain Pruski. 2014. Remote Detection of Mental Workload Changes Using Cardiac Parameters Assessed with a Low-Cost Webcam. Computers in biology and medicine 53 (Oct 2014), 154-163. DOI :

http://dx.doi.org/10.1016/j. compbiomed. 2014.07.014

5. Elissa S Epel, Elizabeth H Blackburn, Jue Lin, Firdaus $S$ Dhabhar, Nancy E Adler, Jason D Morrow, and Richard M Cawthon. 2004. Accelerated Telomere Shortening in Response to Life Stress. Proceedings of the National Academy of Sciences of the United States of America 101, 49 (Dec 2004), 17312-17315. DOI: http://dx.doi.org/10.1073/pnas.0407162101

6. Ericsson Consumerlab. 2015. Living Longer: Wellness and the Internet. Technical Report.

http://www. ericsson. com/res/docs/2015/consumerlab/ consumerlab-living-longer-wellness-and-the-internet. pdf

7. Maurizio Garbarino, Matteo Lai, Daniel Bender, Rosalind W Picard, and Simone Tognetti. 2014. Empatica E3 - a Wearable Wireless Multi-Sensor Device for Real-Time Computerized Biofeedback and Data Acquisition. Proceedings of the 5th EAI International Conference on Wireless Mobile Communication and Healthcare (2014), 39-42. DOI :

http://dx. doi .org/10.1109/MOBIHEALTH. 2014.7015904

8. Javier Hernandez, Pablo Paredes, Asta Roseway, and Mary Czerwinski. 2014. Under Pressure: Sensing Stress of Computer Users. In Proceedings of the SIGCHI Conference on Human Factors in Computing Systems (CHI'14). 51-60. DOI : http://dx.doi.org/10.1145/2556288.2557165

9. Rafal Kocielnik, Natalia Sidorova, Fabrizio Maria Maggi, Martin Ouwerkerk, and Joyce H D M Westerink. 2013. Smart Technologies for Long-Term Stress Monitoring at Work. In Proceedings of the 2013 IEEE 26th International Symposium on Computer-Based Medical Systems (CBMS'13). 53-58. DOI: http://dx.doi.org/10.1109/CBMS. 2013.6627764

10. Robert LiKamWa, Yunxin Liu, Nicholas D Lane, and Lin Zhong. 2013. MoodScope. In Proceeding of the 11th annual international conference (MobiSys '13). 389-402. DOI:http://dx.doi.org/10.1145/2462456.2464449

11. Diana L MacLean, Asta Roseway, and Mary Czerwinski. 2013. MoodWings: a Wearable Biofeedback Device for Real-Time Stress Intervention. PETRA (2013), 66-68. DOI : http://dx.doi .org/10.1145/2504335.2504406
12. Myrthala Moreno-Smith, Susan K Lutgendorf, and Anil K Sood. 2010. Impact of Stress on Cancer Metastasis. Future oncology (London, England) 6, 12 (Dec 2010), 1863-1881. DOI : http://dx.doi.org/10.2217/fon. 10.142

13. Hideyuki Nakashima, Hamid Aghajan, and Juan CarlosEditors Augusto (Eds.). 2010. Handbook of Ambient Intelligence and Smart Environments. Springer US. DOI : http://dx. doi .org/10.1007/978-0-387-93808-0

14. Pascal Paoli and Damien Merllié. Third European survey on working conditions 2000 . Technical Report.

15. Martin Pielot, Tilman Dingler, Jose San Pedro, and Nuria Oliver. 2015. When Attention Is Not Scarce - Detecting Boredom From Mobile Phone Usage. In UbiComp '15: Proceedings of the 2015 ACM International Joint Conference on Pervasive and Ubiquitous Computing. DOI: http://dx.doi .org/10.1145/2750858.2804252

16. Ming-Zher Poh, Nicholas C Swenson, and Rosalind W Picard. 2010. A Wearable Sensor for Unobtrusive, Long-Term Assessment of Electrodermal Activity. IEEE transactions on bio-medical engineering 57, 5 (May 2010), 1243-1252. DOI : http://dx.doi.org/10.1109/TBME. 2009.2038487

17. M T Quazi, S C Mukhopadhyay, N K Suryadevara, and Y M Huang. Towards the smart sensors based human emotion recognition. In 2012 IEEE International Instrumentation and Measurement Technology Conference (I2MTC'12).

18. Carsten Röcker. 2009. Acceptance of Future Workplace Systems: How the Social Situation Influences the Usage Intention of Ambient Intelligence Technologies in Work Environments. In Proceedings of the 2009 International Conference on Work with Computer Systems (WWCS'09).

19. Carsten Röcker. 2010. Services and Applications for Smart Office Environments - a Survey of State-of-the-Art Usage Scenarios. In Proceedings of the 2009 International Conference on Work with Computer Systems (WWCS'09).

20. James A Russell. 1980. A Circumplex Model of Affect. Journal of Personality and Social Psychology 39, 6 (1980), 1161-1178.

21. Pedro Sanches, Kristina Höök, Elsa Vaara, Claus Weymann, Markus Bylund, Pedro Ferreira, Nathalie Peira, and Marie Sjölinder. 2010. Mind the Body!: Designing a Mobile Stress Management Application Encouraging Personal Reflection. In Proceedings of the 8th ACM Conference on Conference on Designing Interactive Systems (DIS '10). 47. DOI: http://dx.doi .org/10.1145/1858171.1858182

22. Holly H Schiffrin and S Katherine Nelson. 2008. Stressed and Happy? Investigating the Relationship Between Happiness and Perceived Stress. Journal of Happiness Studies 11, 1 (Jun 2008), 33-39. DOI : http://dx.doi.org/10.1007/s10902-008-9104-7 
23. Anna Ståhl, Kristina Höök, Martin Svensson, Alex S Taylor, and Marco Combetto. 2009. Experiencing the Affective Diary. Personal and Ubiquitous Computing 13, 5 (2009), 365-378. DOI :

http://dx.doi.org/10.1007/s00779-008-0202-7

24. Andrew Steptoe and Mika Kivimäki. 2012. Stress and Cardiovascular Disease. Nature reviews. Cardiology 9, 6 (Jun 2012), 360-370. DOI :

http://dx.doi.org/10.1038/nrcardio.2012.45

25. Task Force of the European Society of Cardiology and the North American Society of Pacing and Electrophysiology. 1996. Heart Rate Variability: Standards of Measurement, Physiological Interpretation and Clinical Use. Technical Report. Lippincott Williams \& Wilkins. 1043-1065 pages. DOI : http://dx.doi.org/10.1161/01.CIR.93.5.1043

26. The Australian Psychological Society Limited. 2012. Understanding and Managing Stress. Technical Report.

27. Thought Technology Ltd. 2010. Basics of Heart Rate Variability Applied to Psychophysiology. Technical Report.
28. Rui Wang, Fanglin Chen, Zhenyu Chen, Tianxing Li, Gabriella Harari, Stefanie Tignor, Xia Zhou, Dror Ben-Zeev, and Andrew T Campbell. 2014. StudentLife: Assessing Mental Health, Academic Performance and Behavioral Trends of College Students Using Smartphones. In Proceedings of the 2014 ACM International Joint Conference on Pervasive and Ubiquitous Computing (UbiComp '14). ACM Press, 3-14. DOI : http://dx.doi .org/10.1145/2632048.2632054

29. Eli Zelkha, Brian Epstein, Simon Birrel, and Clark Dodsworth. 1998. From Devices to âĂIJAmbient IntelligenceâĂİ. In Digital Living Room Conference.

30. Zhihong Zeng, Maja Pantic, Glenn I Roisman, and Thomas S Huang. A Survey of Affect Recognition Methods: Audio, Visual, and Spontaneous Expressions. IEEE Transactions On Pattern Analysis and Machine Intelligence, IEEE Transactions on 31, 1 (????), 39-58. DOI : http://dx.doi .org/10.1109/TPAMI . 2008.52 